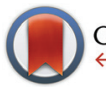

CrossMark

\& click for updates

Cite this: Polym. Chem., 2017, 8, 824

Received 2nd November 2016,

Accepted 20th December 2016

DOI: $10.1039 / \mathrm{c} 6$ py01921h

rsc.li/polymers

\section{Semiconducting alternating multi-block copolymers via a di-functionalized macromonomer approach ${ }^{\dagger}$}

\author{
A. Gasperini, M. Johnson, X. Jeanbourquin, L. Yao, A. Rahmanudin, N. Guijarro and \\ K. Sivula*
}

The development of fully-conjugated semiconducting blockcopolymers is an important goal for organic electronics, but to date has been almost exclusively limited to materials containing poly(3-alkylthiophenes). Here we present the prototype of a class of fully-conjugated semiconducting block copolymers (prepared using a versatile route based on conjugated macromonomers and a cross-coupling polycondensation) that exhibit hole mobility in field effect transistors of the order of $0.1 \mathrm{~cm}^{2} \mathrm{~V}^{-1} \mathrm{~s}^{-1}$ and nanoscopic phase domain separation.

Controlling the self-assembly of $\pi$-conjugated polymers is a key parameter for improving the performance of solutionprocessed semiconducting devices based on organic materials. ${ }^{1-3}$ Fully-conjugated block copolymers have recently emerged as promising materials that combine suitable semiconducting properties with the capability to controllably self-assemble at the nanoscale. ${ }^{4}$ Indeed, the potential ability to change the chemical nature and size of the blocks in a nanophaseseparated copolymer is expected to surmount many of the current challenges in morphology and interfacial structure control for polymer-based electronic devices. For example, in organic photovoltaics (OPVs), which require a nanoscale bulk heterojunction (BHJ) comprised of electron donor and electron acceptor domains, block copolymers offer a route to size-tuneable, thermodynamically stable phase domains. ${ }^{5}$ Moreover, as block copolymers allow for the incorporation of both electron and hole transporting moieties in a single solution processing step, the fabrication of organic light emitting diodes $^{6}$ and OPV devices can be simplified.

To date fully-conjugated block co-polymers have been prepared via step-growth like methods where macromonomers are linked via end-group functionalities, ${ }^{7,8}$ or chain-growth

Laboratory for Molecular Engineering of Optoelectronic Nanomaterials (LIMNO), Institut des Sciences et Ingénierie Chimiques, Ecole Polytechnique Fédérale de Lausanne (EPFL), 1015 Lausanne, Switzerland.E-mail: kevin.sivula@epfl.ch $\dagger$ Electronic supplementary information (ESI) available: Synthesis details and characterization Fig. S1-S6 and Table S1 as described in the main text. See DOI: 10.1039/c6py01921h methods where blocks are grown off the end-functionalized initiators or capped at the end of synthesis. ${ }^{9-13}$ These polymers have demonstrated impressive self-assembly into nanoscale phase domains ${ }^{9,10,14}$ and have indeed been demonstrated as promising materials for use in OPVs. ${ }^{15-18}$

However, in the majority of these examples poly(3-alkylthiophene), most commonly the hexyl derivative, $\mathrm{P} \mathrm{HT},{ }^{19}$ is used for one of the blocks. Indeed, strategies thus far have primarily employed regio-regular P3HT prepared via a nickel-mediated cross-coupling route (McCullough and GRIM methods). This quasi-living route has proved to be central to the synthesis of P3HT with well-defined molecular weights, narrow molecular weight distribution, and-since it affords precise end-group control-for the preparation of macro-monomers for incorporation into block copolymers. ${ }^{10}$ While some recent reports have suggested a method to obtain fully-conjugated block copolymers without $\mathrm{P} 3 \mathrm{HT},{ }^{20-22}$ realizing pure material without a homopolymer has proved to be a challenge. The need to employ P3HT as one of the blocks to obtain pure block copolymers is a clear disadvantage considering that the best performing semiconducting polymer-based devices have matured beyond P3HT employing more sophisticated donor-acceptor type conjugated backbones prepared almost exclusively via stepgrowth polymerization. ${ }^{23}$ To address this disadvantage and open new directions in block copolymer design, the development of synthetic methods that can afford access to a large portfolio of donor or acceptor blocks with tuneable optoelectronic and physical properties is required. ${ }^{4,24}$ Herein, we report a strategy to access a class of fully conjugated alternating multi-block copolymers (MBC) based on step-growth polymerization and preparatory size-exclusion chromatography.

The structure and synthetic scheme for our prototype alternating multi-block copolymer is shown schematically in Fig. 1. For the first block, we chose the well-known poly(bithiophenethienothiophene), pBTTT- $\mathrm{C}_{14}$, given its known ability to selfassemble into ordered domains via the $\pi-\pi$ stacking of the conjugated backbone and linear side chain intercalation, ${ }^{25}$ which we reasoned could aid in the phase segregation of a block copolymer. For the second block we chose a polymer based on 

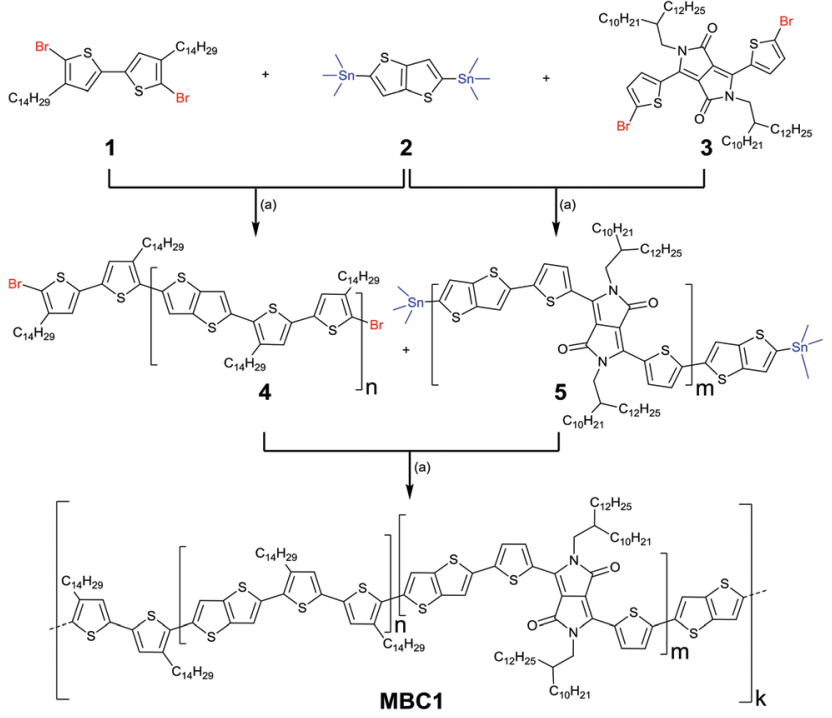

Fig. 1 Synthetic path for the synthesis of the alternating multi-block copolymer MBC. Conditions (a): $\mathrm{Pd}_{2}(\mathrm{dba})_{3}, \mathrm{P}(o-\mathrm{Tol})_{3}$ in dry chlorobenzene for $1 \mathrm{~h}$ at $100^{\circ} \mathrm{C}$.

a DPP-based acceptor ${ }^{26}$ moiety to demonstrate the synthetic versatility of our approach. A standard Stille-coupling polycondensation with a feed stoichiometry imbalance of $1.6: 1$ (of the di-brominated monomer 1 to di-stannylated monomer 2) afforded the di-brominated macromonomer (4) as we have previously shown. ${ }^{27}$ Interestingly, we found this method to also be suitable for preparing di-stannylated macromonomers. Indeed the second block, coded as pDBT-TT (5) with two organotin end groups, was prepared using a reaction feed ratio of $1: 2$ (di-bromo monomer 3 to di-stannylated monomer 2). MALDI-TOF-MS (Fig. S1, ESI $\dagger$ ) shows that the observed peaks are consistent with the molecular weights of the desired difunctionalized macromonomers (see Table S1, ESI $\dagger$ ). We note that while low dispersity $(\nexists=1.2)$ was achieved for pBTTT by the use of preparative size exclusion chromatography (prepSEC), molecular weight range narrowing of block 5 had to be performed through sequential precipitations in acetone and butanone, given the lability of the organotin functionality. Indeed, we found that this end-group could not tolerate slightly acidic polar solvents (i.e. methanol and ethanol) or purification in a standard SEC column. For both di-functionalized blocks, number-average molecular weights were maintained in a specific range ( $5 \mathrm{kDa}<M_{\mathrm{n}}<10 \mathrm{kDa}$ ) in order to guarantee the highest possible reactivity while keeping sufficient chain length to retain the desired optoelectronic characteristics.

The alternating multi-block copolymer (MBC1) was subsequently synthesized using purified 4 (with $M_{\mathrm{n}}=10 \mathrm{kDa}, n \approx 14$ ) and $5\left(M_{\mathrm{n}}=5 \mathrm{kDa}, m \approx 5\right)$. In this case we note that a precise $1: 1$ ratio between functionalities was empirically found to be necessary to achieve reasonable yield. As shown by the analytical size exclusion chromatography (SEC) data (Fig. 2a) of the crude polymerization (grey broken trace), a significant increase in molecular weight was observed from
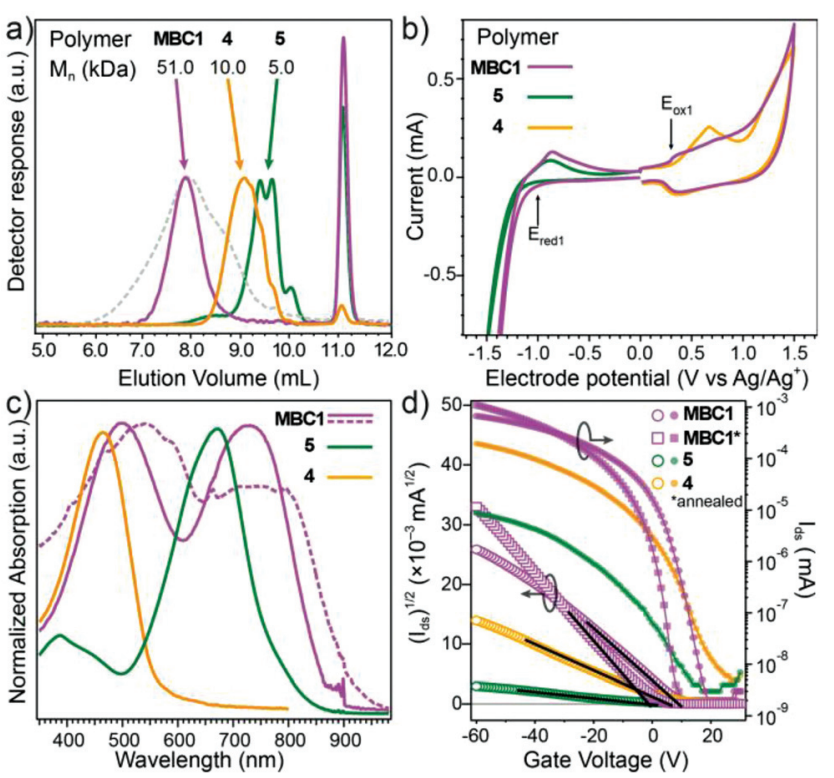

Fig. 2 Characterisation of the prepared MBC1. (a) GPC traces for starting macromonomers 4 and 5 are shown with the crude polymerization product (broken grey trace) and the final purified MBC1. The internal standard peak is also seen at an elution volume of $11 \mathrm{ml}$. (b) CV of MBC1 and the starting materials 4 and 5 recorded at a scan rate of $50 \mathrm{mV} \mathrm{s}^{-1}$ (in $50 \mathrm{mM}$ TBAP in MeCN). (c) Normalized UV-vis absorption spectra of 4, 5, and MBC1 in chlorobenzene (solid traces) and the thin-film solid state absorption of MBC1 (broken trace) after annealing at $200{ }^{\circ} \mathrm{C}$. (d) Transfer characteristics (closed symbols, right) and threshold curves (open symbols, left) from FET devices prepared from as-cast films of 4, 5 , and MBC1, as well as the annealed film of MBC1*.

the starting compounds $\mathbf{4}$ and $\mathbf{5}$. However, a small fraction of unreacted macromonomers remains. The subsequent purification of the crude MBC1 by prep-SEC yielded the final alternating multi-block co-polymer, which exhibited an estimated $M_{\mathrm{n}}$ of $50 \mathrm{kDa}$ with $Ð=1.2(k \approx 6-8)$. The SEC traces confirm that the purified MBC1 is virtually free of unreacted 4 and 5 (less than $5 \%$ overlap). Additional synthetic details and characterization are given in the ESI. $\dagger$ Overall we note that achieving a low dispersity of the macromonomers is critical for the success of our synthetic method as it (i) allows for the accurate determination of end-group feed stoichiometry, (ii) enables the easy verification of the polymerization progression, (iii) facilitates the purification of unreacted macromonomers, and (iv) ultimately simplifies the estimation of the number of blocks incorporated. In addition, it is important to point out that the imbalanced feed stoichiometry route presented here principally allows for choosing the macromonomer end group moiety by swapping the monomer's reactive groups (e.g. 5 could be prepared with DPP end groups by using a di-stannyl version of 3 and a di-bromo 2). This feature is an added advantage given the known importance of the linker group on the optoelectronic properties of the resulting block-copolymer. ${ }^{28}$

In addition to the analytical SEC characterization of MBC1, the incorporation of both blocks was verified by ${ }^{1} \mathrm{H}$-NMR (Fig. S4, ESI $\dagger$ ) where two peaks corresponding to the 
alkyl chains of pDBT-TT and pBTTT segments are observed at $\delta=3.94$ and $2.60 \mathrm{ppm}$ and for the aromatic ring at 8.91 and 7.03-6.88 ppm, respectively. We note that the observed peak areas from the aliphatic protons (both in the alpha position to the aromatic rings) indicate an approximate ratio between the blocks of $1: 2$ (pDBT-TT: pBTTT), which is consistent with the presence of the shorter pDBT-TT ( $5 \mathrm{kDa}$ ) chains compared to the pBTTT (10 kDa). This also implies that an approximately equal number of pDBT-TT and pBTTT blocks are present along the same chain.

To further support the incorporation of both macromonomers in MBC1, its electronic structure (HOMO/LUMO levels) was next estimated by cyclic voltammetry (CV). Fig. 2b illustrates the first oxidation peak $\left(E_{\text {ox1 }}\right)$ of MBC1, which is found at a similar potential to the oxidation peak of neat pBTTT- $\mathrm{C}_{14}$ at $0.20 \mathrm{~V}$ vs. $\mathrm{Ag} / \mathrm{Ag}^{+}(\mathrm{HOMO}=-4.75 \mathrm{eV})$, whereas the reduction peak was found to correspond to the pDBT-TT block at $-1.30 \mathrm{~V} v s . \mathrm{Ag} / \mathrm{Ag}^{+}(\mathrm{LUMO}=-3.55 \mathrm{eV})$. Finally, the UV-Vis absorption spectrum (Fig. 2c) of MBC1 compared to 4 and 5 also gives results consistent with the incorporation of both blocks. In solution, MBC1 (violet trace) shows two characteristic absorption peaks resulting from the weighted contribution of the pBTTT- $\mathrm{C}_{14}$ (yellow) and pDBT-TT block (green) with characteristic $\lambda_{\max }$ at $480 \mathrm{~nm}$ and $700 \mathrm{~nm} .{ }^{16}$ The solidstate thin film absorption spectrum of MBC1 (broken trace) is significantly red-shifted compared to solution, suggesting that the conjugation length or $\pi-\pi$ stacking is enhanced in the solid state, implying some form of self-assembly. Indeed we note that, the red-shift of the peak associated with the pBTTT- $_{14}$ segment is more pronounced and intense compared to the pDBT-TT block.

The effect of covalent attachment of macromonomer blocks on the charge transport in MBC1 was next investigated in thin films by preparing field-effect transistors (FETs) in a bottom contact/bottom-gate configuration. Spin coating the active layer from $o$-DCB solution $\left(10 \mathrm{mg} \mathrm{ml}^{-1}\right)$ afforded film thicknesses of $c a$. 80-100 $\mathrm{nm}$. The transfer curves of representative devices prepared from 4, 5, and MBC1 are shown in Fig. 2d. Interestingly, as-cast devices of MBC1 gave a field-effect hole mobility $\left(\mu_{\mathrm{FET}}\right)$ of $0.05 \mathrm{~cm}^{2} \mathrm{~V}^{-1} \mathrm{~s}^{-1}$ which is comparable with the $\mu_{\mathrm{FET}}$ of non-annealed pBTTT-C $_{14}$ of similar overall $M_{\mathrm{n}}$ $(50 \mathrm{kDa})$ in the same device configuration. ${ }^{29}$ Since thermal annealing of pBTTT- $_{14}$ is routinely performed to increase self-assembly, we annealed MBC1 films at $200{ }^{\circ} \mathrm{C}$ and observed a corresponding increase in the $\mu_{\mathrm{FET}}$ to $0.08 \pm 0.01 \mathrm{~cm}^{2} \mathrm{~V}^{-1} \mathrm{~s}^{-1}$ without changing the on/off ratio $\left(10^{5}\right.$, as seen in the transfer curves). However, an improvement in the FET threshold voltage, $V_{\mathrm{T}}$, is noted with annealing. As shown by the $\left(I_{\mathrm{ds}}\right)^{1 / 2}$ data in Fig. 2d (left, open symbols), $V_{\mathrm{T}}$ decreases from $+7 \mathrm{~V}$ in the as-cast films to $+1 \mathrm{~V}$ after annealing at $200{ }^{\circ} \mathrm{C}$, which is likely due to the improved electrode-active layer contact. $^{30}$ In comparison, the macromonomers $\mathbf{4}$ and $\mathbf{5}$ showed $V_{\mathrm{T}}$ 's close to the value of the unannealed MBC1 and much lower $\mu_{\text {FET }}$ of $9.0 \times 10^{-3} \mathrm{~cm}^{2} \mathrm{~V}^{-1} \mathrm{~s}^{-1}$ and $5.0 \times 10^{-4} \mathrm{~cm}^{2} \mathrm{~V}^{-1} \mathrm{~s}^{-1}$, respectively, likely due to the poor film formation, given their low molecular weight. ${ }^{29}$ Overall the relatively better perform- ance of the FETs prepared with MBC1, the effect of annealing, and the previously discussed UV-vis data strongly indicate that some self-assembly is occurring in the solid state. This is further supported by an increased absorption in the longer wavelength of the film in the solid state after annealing compared to the as-cast state (see Fig. S5, ESI $\dagger$ ).

To gain further insight into the self-assembly of the MBC1 film, we next performed grazing incidence X-ray diffraction (GIXRD). The out-of-plane scattering of the film is shown in Fig. 3a. Macromonomer 5 did not exhibit any peaks (data not shown), as expected, due to its short length, considering that the crystallinity of this polymer has been shown to decrease with decreasing $M_{\mathrm{n}},{ }^{31}$ possibly due to the different self-assembly of the macromonomers with a different number of repeating units. However, the pBTTT block 4 showed the expected (h00) peaks due to lamellar stacking with a $d$-spacing of $21.0 \AA$ A. MBC1 also showed lamellar stacking peaks, but with a slightly expanded $d$-spacing of $22.4 \AA$ A. This difference could be due to the larger branched alkyl chains on the pDBT-TT block, which force a greater equilibrium stacking distance. Atomic force microscopy (AFM) performed in tapping mode on the surface of an annealed thin film of MBC1 further revealed a "fingerprint-like" nanophase-separated morphology as shown by the phase image in Fig. 3b. We note that the features observed are distinct from variations in film height (see the height trace image in Fig. S6, ESI†) suggesting that the features are due to chemical differences in the film. The integrated radial profile of the fast Fourier transform of the AFM phase image yields an amplitude plot versus the spatial frequency, $\xi$ (Fig. 3c), which quantifies the periodicity of the observed features. We note that a peak at $\xi=0.005$ cycles per $\mathrm{nm}$ (corresponding to $200 \mathrm{~nm}$ features) is a result of the large dark circular features visible in the topography which are associated with height depressions in the film (see Fig. S6, ESI $\dagger$ ). In addition, a clear peak at $\xi=0.021$ cycles per nm corresponds to a crest-to-crest domain size of $c a .50 \mathrm{~nm}$ for the main block copolymer features. Although there is a slight dispersion in the block length $(\nexists=1.2)$, the length of the $10 \mathrm{kDa}$ pBTTT used to prepare MBC1 would, by itself, exhibit ca. $40 \mathrm{~nm}$ wide domains in the "fibril-type" morphology. ${ }^{29}$ Thus the observed morphology in the MBC1 film together with the XRD results, suggest that domains of straight, $\pi-\pi$ stacked and pBTTT chains are self-assembled and separated by amorphous pDBT-TT
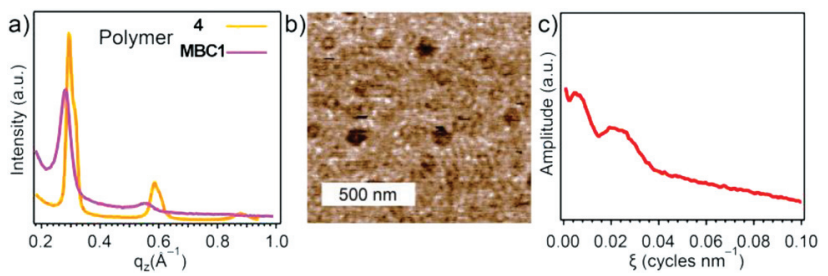

Fig. 3 Characterization of the self-assembly of MBC1. (a) Shows the out-of-plane (z-direction) grazing-incidence X-ray diffractogram of a thin film of MBC1 on a $\mathrm{SiO}_{2}$ substrate. (b) Shows the phase image from a tapping mode AFM image, and (c) shows the amplitude of the spatial frequency from the FFT of (b) as described in the main text. 
domains of smaller relative volume given their $2: 1$ ratio of pBTTT : pDBT-TT blocks. This gives a first clear indication that the multi-block co-polymer approach introduced here can effectively yield materials capable of nanoscale phase segregation.

In summary, we have demonstrated a straightforward approach to prepare fully-conjugated alternating multi-block copolymers for optoelectronic applications. Our synthetic strategy relies on stoichiometry control and purification to prepare precise di-functionalized macromonomers, prep-SEC to isolate the final multi-block copolymer, and allows broad selection of block chemistry. Standard characterization of the resulting MBC1 polymer confirmed the presence of both blocks and demonstrated good charge transport as the active layer in a transistor. Moreover, morphological characterization suggests the ability of our prototype polymer to self-assemble into nano-scale domains. Our approach is thus promising for further design of the next generation of semiconducting polymers with thermodynamically stable electronic properties that are fully tunable by changing the block structure and length. Indeed, the ability to precisely control the size of n- and p-type domains in thermodynamically stable bulk-heterojunctions at the length scale of the exciton transport $(\sim 10 \mathrm{~nm})$ holds the key to the development of next generation organic solar cells ${ }^{24}$ and organic light emitting diodes. ${ }^{32}$

This work was partly supported by the Swiss National Science Foundation (Project No. 200021 169215) and the European Research Council (Project No. 336506, CEMOS).

\section{Notes and references}

1 Y. Diao, L. Shaw, Z. Bao and S. C. B. Mannsfeld, Energy Environ. Sci., 2014, 7, 2145-2159.

2 F. H. Schacher, P. A. Rupar and I. Manners, Angew. Chem., Int. Ed., 2012, 51, 7898-7921.

3 S. B. Darling, Energy Environ. Sci., 2009, 2, 1266-1273.

4 M. J. Robb, S.-Y. Ku and C. J. Hawker, Adv. Mater., 2013, 25, 5686-5700.

5 H. Benten, D. Mori, H. Ohkita and S. Ito, J. Mater. Chem. A, 2016, 4, 5340-5365.

6 R. A. Segalman, B. McCulloch, S. Kirmayer and J. J. Urban, Macromolecules, 2009, 42, 9205-9216.

7 D. Izuhara and T. M. Swager, Macromolecules, 2011, 44, 2678-2684.

8 K. B. Woody, B. J. Leever, M. F. Durstock and D. M. Collard, Macromolecules, 2011, 44, 4690-4698.

9 R. Verduzco, I. Botiz, D. L. Pickel, S. M. Kilbey, K. Hong, E. Dimasi and S. B. Darling, Macromolecules, 2011, 44, 530539.

10 S.-Y. Ku, M. A. Brady, N. D. Treat, J. E. Cochran, M. J. Robb, E. J. Kramer, M. L. Chabinyc and C. J. Hawker, J. Am. Chem. Soc., 2012, 134, 16040-16046.
11 R. C. Mulherin, S. Jung, S. Huettner, K. Johnson, P. Kohn, M. Sommer, S. Allard, U. Scherf and N. C. Greenham, Nano Lett., 2011, 11, 4846-4851.

12 Y.-H. Lin, K. A. Smith, C. N. Kempf and R. Verduzco, Polym. Chem., 2013, 4, 229-232.

13 C. Pan, K. Sugiyasu, J. Aimi, A. Sato and M. Takeuchi, Angew. Chem., Int. Ed., 2014, 53, 8870-8875.

14 M. Knaapila, T. Costa, V. M. Garamus, M. Kraft, M. Drechsler, U. Scherf and H. D. Burrows, J. Phys. Chem. $B, 2015,119,3231-3241$.

15 C. Guo, Y.-H. Lin, M. D. Witman, K. A. Smith, C. Wang, A. Hexemer, J. Strzalka, E. D. Gomez and R. Verduzco, Nano Lett., 2013, 13, 2957-2963.

16 J. W. Mok, Y.-H. Lin, K. G. Yager, A. D. Mohite, W. Nie, S. B. Darling, Y. Lee, E. Gomez, D. Gosztola, R. D. Schaller and R. Verduzco, Adv. Funct. Mater., 2015, 25, 5578-5585.

17 S. Wang, Q. Yang, Y. Tao, Y. Guo, J. Yang, Y. Liu, L. Zhao, Z. Xie and W. Huang, New J. Chem., 2016, 40, 1825-1833.

18 F. Lombeck, A. Sepe, R. Thomann, R. H. Friend and M. Sommer, ACS Nano, 2016, 10, 8087-8096.

19 Y.-H. Lin, K. G. Yager, B. Stewart and R. Verduzco, Soft Matter, 2014, 10, 3817-3825.

20 T. Yokozawa, H. Kohno, Y. Ohta and A. Yokoyama, Macromolecules, 2010, 43, 7095-7100.

21 D. Gao, G. L. Gibson, J. Hollinger, P. Li and D. S. Seferos, Polym. Chem., 2015, 6, 3353-3360.

22 J. W. Mok, D. Kipp, L. R. Hasbun, A. Dolocan, J. Strzalka, V. Ganesan and R. Verduzco, J. Mater. Chem. A, 2016, 4, 14804-14813.

23 C. Liu, K. Wang, X. Gong and A. J. Heeger, Chem. Soc. Rev., 2016, 45, 4825-4846.

24 Y. Lee and E. D. Gomez, Macromolecules, 2015, 48, 73857395.

25 M. L. Chabinyc, M. F. Toney, R. J. Kline, I. McCulloch and M. Heeney, J. Am. Chem. Soc., 2007, 129, 3226-3237.

26 W. Li, K. H. Hendriks, W. S. C. Roelofs, Y. Kim, M. M. Wienk and R. A. J. Janssen, Adv. Mater., 2013, 25, 3182-3186.

27 A. Gasperini, S. Bivaud and K. Sivula, Chem. Sci., 2014, 5, 4922-4927.

28 K. Johnson, Y.-S. Huang, S. Huettner, M. Sommer, M. Brinkmann, R. Mulherin, D. Niedzialek, D. Beljonne, J. Clark, W. T. S. Huck and R. H. Friend, J. Am. Chem. Soc., 2013, 135, 5074-5083.

29 A. Gasperini and K. Sivula, Macromolecules, 2013, 46, 93499358.

30 J. F. Martínez Hardigree and H. E. Katz, Acc. Chem. Res., 2014, 47, 1369-1377.

31 A. Gasperini, X. A. Jeanbourquin and K. Sivula, J. Polym. Sci., Part B: Polym. Phys., 2016, 54, 2245-2253.

32 Y. Chen, J. Chen, D. Ma, D. Yan, L. Wang and F. Zhu, Appl. Phys. Lett., 2011, 98, 243309. 